\title{
The Moment Method Solution for Printed Wire Antennas of Arbitrary Configuration
}

\author{
HISAMATSU NAKANO, SENIOR MEMBER, IEEE, STEPHEN R. KERNER, STUDENT MEMBER, IEEE, AND \\ NICÓLAOS G. ALEXOPOULOS, FELLOW, IEEE
}

\begin{abstract}
The analysis of a printed wire antenna of arbitrary configuration is presented. The electric field tangential to the wire is derived using the current expanded by piecewise sinusoidal functions. These functions are also used to form the impedance matrix elements. Use of the stationary phase method leads to a simple expression for the radiation field. The numerical analyses based on the present formulation reveal the radiation characteristics of a zigzag dipole antenna, a loop antenna, and a round spiral antenna.
\end{abstract}

\section{INTRODUCTION}

$\mathrm{W}$ IRE RADIATING elements, such as linear dipoles or loops [1], are found (with or without a conducting plane reflector) in many practical antennas. In most cases, analyses of these elements have assumed that they are located in free space. Also, numerical solutions are usually based on either Pocklington-type [2] or Halien-type [3] integral equations, and normally are not designed to handle antennas in a space partially filled with dielectric material.

It is well known to antenna engineers that a dielectric material may be used to provide support for practical wire antennas, and that a conducting plane reflector is a simple mechanism for obtaining a unidirectional radiation beam. Consequently, both a dielectric and a reflector are often incorporated into a printed circuit antenna design. Two approaches provide rigorous solutions to this type of problem: the spectral domain method [4]-[6] and the real-axis numerical integration method [7], [8] used in this paper. Additional examples of the real-axis treatment, which is characterized by Sommerfeld-type integrals and moment-method solutions, can be found in [9]-[11].

The motivation for this research comes about because the technical literature, of which [4]-[11] are typical, refers only to linear configurations; a generalized treatment of a curved or bent antenna on a grounded substrate has not been developed. Our attention, therefore, is focused on a printed wire antenna of arbitrary configuration.

The first goal is to develop an equation for the tangential electric field. Application of the method of moments yields an impedance matrix, which may be solved for the current distribution and other properties. After deriving an expression for the far-zone radiation field, which is simplified by the

Manuscript received August 14, 1987; revised April 8, 1988

H. Nakano was with the University of California, Los Angeles, CA. He is now with the College of Engineering, Hosei University, Koganei City, Tokyo 184, Japan.

S. R. Kerner and N. G. Alexopoulos are with the Electrical Engineering Department, University of California, Los Angeles, CA 90024

IEEE Log Number 8823635 stationary phase method, we illustrate the power of the formulation by analyzing three types of antennas: a zigzag dipole, a loop, and a round spiral. The antenna characteristics are determined by the computed current distributions and the particular geometry of each antenna. The present formulation makes it possible to analyze almost all kinds of linear-, bent-, and curved-type printed wire antennas.

\section{Determination of Current Distribution}

Fig. 1(a) shows a printed wire antenna (rather than a printed strip antenna) of arbitrary configuration mounted on a grounded dielectric substrate of thickness $B$ and relative permittivity $\epsilon_{r}$. The assumptions for the formulation are as follows.

1) The wire radius $a$ is small enough for the thin wire approximation to be applied; that is, $a \ll \lambda_{0}$, where $\lambda_{0}$ is the free-space wavelength.

2) Only the axial component of the current contributes to the antenna characteristics, such as the input impedance and the radiation pattern. The nonsymmetrical circumferential component may be neglected under the conditions of assumption 1 [11].

3) The antenna arm is subdivided into many segments which may be regarded as linear elements. This assumption is often used for the analysis of curved wire antennas, such as loops and spirals [12].

We shall express the net current on the segments in the form $I(X)=\sum I_{n} J_{n}(X)$, where the $J_{n}$ are expansion functions, and the $I_{n}$ are coefficients to be determined. As shown in Fig. 2(a), piecewise sinusoids are chosen as the expansion functions in this paper:

$$
J_{n}(X)= \begin{cases}\frac{\sin k\left(X-X_{n-1}\right)}{\sin k d}, & X_{n-1} \leq X \leq X_{n} \\ \frac{\sin k\left(X_{n+1}-X\right)}{\sin k d}, & X_{n} \leq X \leq X_{n+1} \\ 0, & \text { otherwise }\end{cases}
$$

where $X, X_{n-1}$, and $X_{n}$ are the distances measured from arm end to points along the antenna arm. These distances should be distinguished from the local distances or coordinates $x^{\prime}$ and $t$ which soon appear.

This piecewise sinusoidal formulation requires three steps 


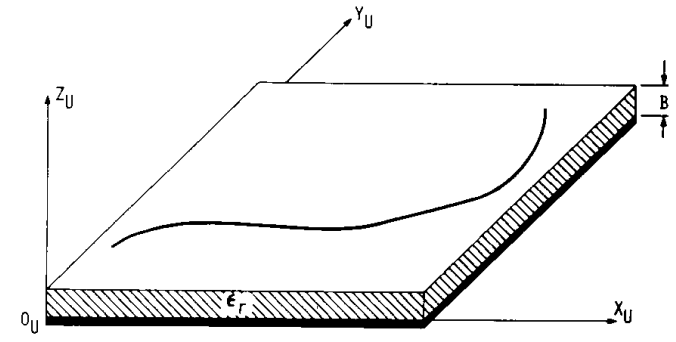

(a)

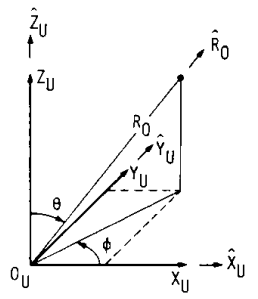

(b)

Fig. 1. Printed antenna of arbitrary configuration. $\left(\hat{X}_{u}, \hat{Y}_{u}, \hat{Z}_{u}\right.$, and $\hat{R}_{0}$ are unit vectors.)
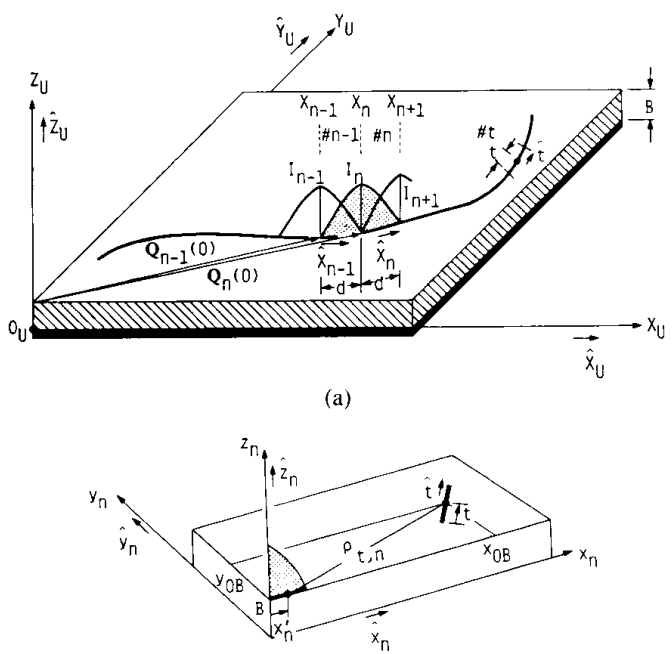

(b)

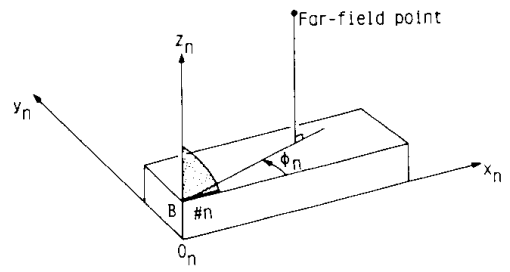

(c)

Fig. 2. Piecewise sinusoidal expansion function.

to determine the current distribution. These are derivations of the tangential electric field due to a single element, the tangential field due to all elements, and the impedance matrix formation by a reaction process.

1) We begin with a single element of length $d$ picked from Fig. 2(a). This is shown in Fig. 3. The electric field e due to

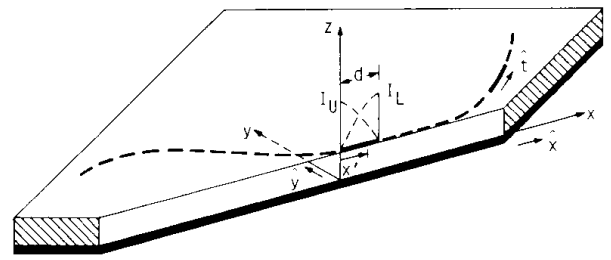

Fig. 3. Single element from structure of Fig. 2.

the current $I\left(x^{\prime}\right) \hat{\mathbf{x}}$ on this element is expressed as follows:

$$
\mathbf{e}=\int_{0}^{d}\left\{\left(k^{2} \overline{\overline{\mathbf{I}}}+\nabla \nabla\right) \cdot \overline{\bar{\Pi}}\right\} \cdot I\left(x^{\prime}\right) \hat{\mathbf{x}} d x^{\prime}
$$

where $\overline{\overline{\mathbf{I}}}=\hat{\mathbf{x}} \hat{\mathbf{x}}+\hat{\mathbf{y}} \hat{\mathbf{y}}+\hat{\mathbf{z}} \hat{\mathbf{z}}, k=\omega \sqrt{\mu_{0} \epsilon_{0}}$, and $\overline{\bar{\Pi}}$ is the dyadic Hertz vector potential function. Note that the time dependence $\exp (+j \omega t)$ is omitted throughout the paper.

We write the current distribution on the single element as

$$
I\left(x^{\prime}\right)=I_{L} \frac{\sin k x^{\prime}}{\sin k d}+I_{U} \frac{\sin k\left(d-x^{\prime}\right)}{\sin k d}
$$

where $I_{L}$ and $I_{U}$ are temporary expressions for the expansion function coefficients. By substituting (3) into (2) and taking the inner product with a unit vector $\hat{\mathbf{t}}$ tangential to the element on which an observation point $(x, y, z=B)$ is located, the tangential component of the electric field at this observation point can be obtained

$$
e_{t}(x, y, B)=I_{L} \frac{1}{\sin k d}(\mathbf{L} \cdot \hat{\mathbf{t}})+I_{U} \frac{1}{\sin k d}(\mathbf{U} \cdot \hat{\mathbf{t}})
$$

where

$$
\begin{gathered}
\mathbf{L}=\int_{0}^{d}\left(\Gamma^{x} \hat{\mathbf{x}}+\Gamma^{y} \hat{\mathbf{y}}\right) \sin k x^{\prime} d x^{\prime} \\
\mathbf{U}=\int_{0}^{d}\left(\Gamma^{x} \hat{\mathbf{x}}+\Gamma^{y} \hat{\mathbf{y}}\right) \sin k\left(d-x^{\prime}\right) d x^{\prime} .
\end{gathered}
$$

In the above equations, $\Gamma^{x}$ and $\Gamma^{y}$ obey the following definitions:

$$
\begin{gathered}
\Gamma^{x}=\frac{\partial^{2} \Pi^{x}}{\partial x^{2}}+\frac{\partial^{2} \Pi^{z}}{\partial x \partial z}+k^{2} \Pi^{x} \\
\Gamma^{y}=\frac{\partial^{2} \Pi^{x}}{\partial y \partial x}+\frac{\partial^{2} \Pi^{z}}{\partial y \partial z}
\end{gathered}
$$

where $\Pi^{x}$ and $\Pi^{z}$ are Sommerfeld-type integrals and are obtained by applying boundary conditions at the air-dielectric interface $Z=B$ :

$$
\Pi^{x}=2 u \lim _{z \rightarrow B} \int_{0}^{\infty} J_{0}(\rho \lambda) e^{-\mu(z-B)} \frac{\lambda}{D_{e}(\lambda)} d \lambda
$$

$\Pi^{z}=2 u\left(1-\epsilon_{r}\right) \lim _{z \rightarrow B}$

$$
\cdot \int_{0}^{\infty} \cos \phi J_{1}(\rho \lambda) e^{-\mu(z-B)} \frac{\lambda^{2}}{D_{e}(\lambda) D_{m}(\lambda)} d \lambda \text {. }
$$


In these equations (and (16) below), originally derived by Rana [11], $\rho$ is the distance between the source point $\left(x^{\prime}, y^{\prime}\right.$, $B)$ and the observation point $(x, y, B), \cos \phi=\left(x-x^{\prime}\right) / \rho$, $u=-j /\left(4 \pi \epsilon_{0} \omega\right)$, and

$$
\begin{gathered}
D_{e}(\lambda)=\mu+\mu_{e} \operatorname{coth} \mu_{e} B \\
D_{m}(\lambda)=\mu \epsilon_{r}+\mu_{e} \tanh \mu_{e} B
\end{gathered}
$$

with

$$
\mu=\sqrt{\lambda^{2}-k^{2}}
$$

and

$$
\mu_{e}=\sqrt{\lambda^{2}-\epsilon_{r} k^{2}}
$$

From (5), (7) and (8), the inner product $\mathbf{L} \cdot \hat{\mathbf{t}}$ is expressed as

$$
\begin{aligned}
\mathbf{L} \cdot \hat{\mathbf{t}}= & \int_{0}^{d}\left\{\frac{\partial}{\partial x} \frac{\partial \Pi^{x}}{\partial x}(\hat{\mathbf{x}} \cdot \hat{\mathbf{t}})+\frac{\partial}{\partial y} \frac{\partial \Pi^{x}}{\partial x}(\hat{\mathbf{y}} \cdot \hat{\mathbf{t}})\right\} \sin k x^{\prime} d x^{\prime} \\
& +\int_{0}^{d}\left\{\frac{\partial}{\partial x} \frac{\partial \Pi}{\partial x^{\prime}}(\hat{\mathbf{x}} \cdot \hat{\mathbf{t}})+\frac{\partial}{\partial y} \frac{\partial \Pi}{\partial x^{\prime}}(\hat{\mathbf{y}} \cdot \hat{\mathbf{t}})\right\} \\
& \cdot \sin k x^{\prime} d x^{\prime} \\
& +(\hat{\mathbf{x}} \cdot \hat{\mathbf{t}}) \int_{0}^{d} k^{2} \Pi^{x} \sin k x^{\prime} d x^{\prime}
\end{aligned}
$$

where $\Pi$ is defined as

$$
\Pi=2\left(\epsilon_{r}-1\right) u \lim _{z \rightarrow B} \int_{0}^{\infty} J_{0}(\rho \lambda) e^{-\mu(z-B)} \frac{\lambda \mu}{D_{e}(\lambda) D_{m}(\lambda)} d \lambda
$$

The treatment of the integrands of (9) and (16) is addressed in the literature [13].

Using an expression for the derivative with respect to the coordinate $t$, which is the distance between the observation point and the beginning of the observation point segment, we may simplify (15) as

$$
\begin{aligned}
\mathbf{L} \cdot \hat{\mathbf{t}}= & \int_{0}^{d} \frac{d}{d t}\left(\frac{\partial \Pi^{x}}{\partial x}+\frac{\partial \Pi}{\partial x^{\prime}}\right) \sin k x^{\prime} d x^{\prime} \\
& +(\hat{\mathbf{x}} \cdot \hat{\mathbf{t}}) \int_{0}^{d} k^{2} \Pi^{x} \sin k x^{\prime} d x^{\prime}
\end{aligned}
$$

Similarly, the inner product $\mathbf{U} \cdot \hat{\mathbf{t}}$ in (4) defined by (6)-(8) is simplified to

$$
\begin{aligned}
\mathbf{U} \cdot \hat{\mathbf{t}}= & \int_{0}^{d} \frac{d}{d t}\left(\frac{\partial \Pi^{x}}{\partial x}+\frac{\partial \Pi}{\partial x^{\prime}}\right) \sin k\left(d-x^{\prime}\right) d x^{\prime} \\
& +(\hat{\mathbf{x}} \cdot \hat{\mathbf{t}}) \int_{0}^{d} k^{2} \Pi^{x} \sin k\left(d-x^{\prime}\right) d x^{\prime}
\end{aligned}
$$

2) Based on the results for the single element, an electric field due to a pair of elements labeled $n-1$ and $n$, as shown in Fig. 2(a), is now considered. In Fig. 2(a), the observation point is located on the element labeled $t$. We adopt the local coordinate systems $\left(x_{n-1}, y_{n-1}, z_{n-1}\right)$ and $\left(x_{n}, y_{n}, z_{n}\right)$ for simplicity, in which the corresponding unit vectors are defined as $\left(\hat{\mathbf{x}}_{n-1}, \hat{\mathbf{y}}_{n-1}, \hat{\mathbf{z}}_{n-1}\right)$ and $\left(\hat{\mathbf{x}}_{n}, \hat{\mathbf{y}}_{n}, \hat{\mathbf{z}}_{n}\right)$, as shown in Fig. 2(b). The $x$-coordinate is taken in such a way that it is parallel to the element axis.

The tangential component of the electric field from the two elements $n-1$ and $n$ is derived from (4), with $I_{L}=I_{U}=I_{n}$ :

$$
e_{n, t}=I_{n}\left(\mathbf{e}_{n} \cdot \hat{\mathbf{t}}\right)
$$

where

$$
\mathbf{e}_{n} \cdot \hat{\mathbf{t}}=\frac{1}{\sin k d}\left(\mathbf{L}_{n-1} \cdot \hat{\mathbf{t}}+\mathbf{U}_{n} \cdot \hat{\mathbf{t}}\right)
$$

in which we have made use of (17) and (18) for $\mathbf{L}_{n-1} \cdot \hat{\mathbf{t}}$ and $\mathbf{U}_{n} \cdot \hat{\mathbf{t}}$, adding additional subscripts $n-1$ and $n$, respectively:

$$
\begin{aligned}
\mathbf{L}_{n-1} \cdot \hat{\mathbf{t}}= & \int_{0}^{d} \frac{d}{d t}\left(\frac{\partial G_{t, n-1}}{\partial x_{n-1}^{\prime}}\right) \sin k x_{n-1}^{\prime} d x_{n-1}^{\prime} \\
& +\left(\hat{\mathbf{x}}_{n-1} \cdot \hat{\mathbf{t}}\right) \int_{0}^{d} k^{2} \Pi_{t, n-1}^{x} \sin k x_{n-1}^{\prime} d x_{n-1}^{\prime} \\
\mathbf{U}_{n} \cdot \hat{\mathbf{t}}= & \int_{0}^{d} \frac{d}{d t}\left(\frac{\partial G_{t, n}}{\partial x_{n}^{\prime}}\right) \sin k\left(d-x_{n}^{\prime}\right) d x_{n}^{\prime} \\
& +\left(\hat{\mathbf{x}}_{n} \cdot \hat{\mathbf{t}}\right) \int_{0}^{d} k^{2} \Pi_{t, n}^{x} \sin k\left(d-x_{n}^{\prime}\right) d x_{n}^{\prime}
\end{aligned}
$$

where $G_{t, n-1}$ and $G_{t, n}$ obey the following definition:

$$
G_{t, j}=-\Pi_{t, j}^{x}+\Pi_{t, j}, \quad j=n-1, n .
$$

Note that $\Pi^{x}$ and $\Pi$ from (9) and (16) are emphasized in (23) by subscripts $t$ and $j$, which indicate that the observation point and source point are located on the elements labeled $t$ and $j(=n-1, n)$, respectively. These subscripts show that the distance $\rho$ in (9) and (16) is given as $\rho \equiv \rho_{t, j}=\left\{\left(x_{\mathrm{OB}}-x_{j}^{\prime}\right)^{2}\right.$ $\left.+y_{\mathrm{OB}}^{2}\right\}^{1 / 2}$, in which the position of the observation point $\left(x_{\mathrm{OB}}, y_{\mathrm{OB}}\right)$ is measured using the local coordinate system of the $j$ th element $(j=n-1, n)$. Also, note that in (21) and (22) the relation $\partial \Pi_{t, j}^{x} / \partial x_{j}=-\partial \Pi_{t, j}^{x} / \partial x_{j}^{\prime}$ has been used.

Summation of (19) over all currents gives us the tangential component of the total electric field, which we write as

$$
\begin{aligned}
E_{\mathrm{tan}}=\frac{1}{\sin k d} \sum_{n} I_{n}\left\{\int_{0}^{d} N_{t, n-1} \sin k x_{n-1}^{\prime} d x_{n-1}^{\prime}\right. \\
\left.\quad+\int_{0}^{d} N_{t, n} \sin k\left(d-x_{n}^{\prime}\right) d x_{n}^{\prime}\right\}
\end{aligned}
$$

where

$$
N_{t, j}=\frac{d}{d t}\left(\frac{\partial G_{t, j}}{\partial x_{j}^{\prime}}\right)+k^{2}\left(\hat{\mathbf{x}}_{j} \cdot \hat{\mathbf{t}}\right) \Pi_{t, j}^{x}, \quad j=n-1, n .
$$

3) The final stage to obtain the current distribution is completed by application of weighting functions to (24). This 
process gives us a set of linear equations:

$$
[V]=[Z][I]
$$

where $[V],[Z]$, and $[I]$ are the voltage, impedance, and current matrices, respectively, for the moment method. The unknown currents $[I]$ of (26) are then determined by standard techniques.

With testing functions defined as in (1), the impedance matrix element $Z_{m, n}$ is calculated by

$$
\begin{aligned}
Z_{m, n}=\int_{0}^{d} \frac{\sin k x_{m-1}}{\sin k d} \mathbf{e}_{n} & \cdot \hat{\mathbf{x}}_{m-1} d x_{m-1} \\
& +\int_{0}^{d} \frac{\sin k\left(d-x_{m}\right)}{\sin k d} \mathbf{e}_{n} \cdot \hat{\mathbf{x}}_{m} d x_{m}
\end{aligned}
$$

where the local distances $x_{m-1}$ and $x_{m}$ are defined as equal to $X-X_{m-1}$ and $X-X_{m}$, respectively. We can calculate $\left(\mathbf{e}_{n} \cdot \hat{\mathbf{x}}_{m-1}\right)$ and $\left(\mathbf{e}_{n} \cdot \hat{\mathbf{x}}_{m}\right)$ from (20), with $\hat{\mathbf{t}}=\hat{\mathbf{x}}_{m-1}$ and $\hat{\mathbf{t}}=\hat{\mathbf{x}}_{m}$, respectively. The overlapping of source and observation points, which appears in the self-impedance terms for $m=n$, is avoided by using a wire radius.

\section{Evaluation of Antenna Characteristics}

The antenna characteristics are calculated using the obtained current distribution. For a delta-gap source excitation, we may evaluate the input impedance as $Z_{\text {in }}=V / I_{\text {in }}$, where $I_{\text {in }}$ is the current flowing between the terminals where the small gap is excited with an input voltage $V$.

As seen from (2), the radiation pattern at a far-field point $\left(R_{o}, \theta, \phi\right)$ shown in Fig. 1(b) might be directly calculated by integration of the obtained current distribution with the Hertz vector potential function. This is quite time consuming, and hence we use the stationary phase method [14] to calculate the radiation field. The result for the electric field from the elements $n-1$ and $n$ is expressed as

$$
I_{n} \mathbf{e}_{f, n}=I_{n} n_{f} e^{j k B \cos \theta}\left(f_{\theta, n} \hat{\theta}+f_{\phi, n} \hat{\phi}\right)
$$

where $n_{f}$ is $(u k) \exp \left(-j k R_{o}\right) /\left(R_{o} \sin k d\right)$, and

$$
\begin{gathered}
f_{\theta, n}=\frac{\theta(\theta, B)}{D_{e}(\theta, B)}\left(-\alpha_{n-1} \cos \phi_{n-1}+\alpha_{n} \cos \phi_{n}\right) \\
f_{\phi, n}=\frac{1}{D_{e}(\theta, B)}\left(\alpha_{n-1} \sin \phi_{n-1}-\alpha_{n} \sin \phi_{n}\right)
\end{gathered}
$$

in which

$$
\begin{gathered}
\theta(\theta, B)=\cos \theta+\frac{\epsilon_{r}-1}{\epsilon_{r}} \frac{\tan \theta \sin \theta}{D_{m}(\theta, B)} \\
D_{e}(\theta, B)=1-j \frac{\sqrt{\epsilon_{r}-\sin ^{2} \theta}}{\cos \theta} \cot \left(k B \sqrt{\epsilon_{r}-\sin ^{2} \theta}\right) \\
D_{m}(\theta, B)=1+j \frac{\sqrt{\epsilon_{r}-\sin ^{2} \theta}}{\epsilon_{r} \cos \theta} \tan \left(k B \sqrt{\epsilon_{r}-\sin ^{2} \theta}\right)
\end{gathered}
$$

$$
\begin{aligned}
& \alpha_{i}=\left(A_{i} \mathbf{e}^{-j k d \delta_{i, n}}+B_{i} \mathbf{e}^{+j k d \delta_{i, n}}\right) \exp \left\{j k \mathbf{Q}_{i}(0) \cdot \hat{\mathbf{R}}_{0}\right\}, \\
& i=n-1, n ; \delta_{n-1, n}=0, \delta_{n, n}=1 \\
& A_{i}=\frac{\exp \left[+j k d\left(1+\sin \theta \cos \phi_{i}\right)\right]-1}{1+\sin \theta \cos \phi_{i}}, \quad i=n-1, n \\
& B_{i}=\frac{\exp \left[-j k d\left(1-\sin \theta \cos \phi_{i}\right)\right]-1}{1-\sin \theta \cos \phi_{i}}, \quad i=n-1, n \text {. }
\end{aligned}
$$

As defined in Fig. 2(a), $\mathbf{Q}_{i}(0)$ in (34) is a vector directed from a point $\left(X_{U}=0, Y_{U}=0, Z_{U}=B\right)$ of the universal coordinate system to the starting point of the $i$ th element, where $i=n-1$ or $i=n$. In (35) and (36), $\phi_{i}$ is an angle between the axis of the $i$ th element (parallel to the $x_{i}$ axis) and a line joining the starting point of this element with the projection of the far-field point into the universal $X_{U}-Y_{U}$ plane at $Z_{U}=B$ (see Fig. 2(c)). Therefore,

$$
\begin{gathered}
\cos \phi_{i}=\cos \phi\left(\hat{\mathbf{X}}_{U} \cdot \hat{\mathbf{x}}_{i}\right)+\sin \phi\left(\hat{\mathbf{Y}}_{U} \cdot \hat{\mathbf{x}}_{i}\right) \\
\sin \phi_{i}=-\cos \phi\left(\hat{\mathbf{Y}}_{U} \cdot \hat{\mathbf{x}}_{i}\right)+\sin \phi\left(\hat{\mathbf{X}}_{U} \cdot \hat{\mathbf{x}}_{i}\right)
\end{gathered}
$$

where $\hat{\mathbf{X}}_{U}$ and $\hat{\mathbf{Y}}_{U}$ are the unit vectors for the universal coordinates $X_{U}$ and $Y_{U}$.

Summing (28) over all elements, we have $\mathbf{E}_{f}$, the total radiation field from the antenna

$$
\mathbf{E}_{f}=n_{f} e^{j k B \cos \theta}\left(F_{\theta} \hat{\theta}+F_{\phi} \hat{\phi}\right)
$$

where

$$
F_{i}=\sum_{n} I_{n} f_{i, n}, \quad i=\theta, \phi
$$

\section{Numerical Results}

A computer program based on the results of Sections II and III reproduced the published data of a linear dipole on dielectric materials [11], a zigzag antenna [15], and a spiral antenna [12], the latter two being in free space. The former linear dipole and the latter two antennas were used for checking the validity of the computer program regarding the presence of the dielectric materials and the treatment of bent or curved configurations, respectively. These preliminary checks were successful.

We analyze three antennas in this section. Each antenna is printed on a grounded substrate of thickness $B=0.1016 \lambda_{0}$. The printed wires have a radius of $10^{-4} \lambda_{0}$, and form configurations of a zigzag dipole [15], a circular loop [1], and a round spiral [12]. The permittivity for the first two cases is $\epsilon_{\text {, }}$ $=2.000$, while the round spiral uses $\epsilon_{r}=6.055$.

It is worth mentioning the resonance of a zigzag dipole antenna. This resonance is observed at a bend angle of about $120^{\circ}$, as shown in Fig. 4. This antenna has the lengths of the zigzag elements $e_{1}=e_{7}=0.0167 \lambda_{0}$ and $e_{2}=e_{3}=\cdots=$ $e_{6}=0.0333 \lambda_{0}$, resulting in the total wire length $2 L_{\text {wire }}=0.4$ $\lambda_{0}$. The shortening ratio (SR) relative to the half-wavelength 


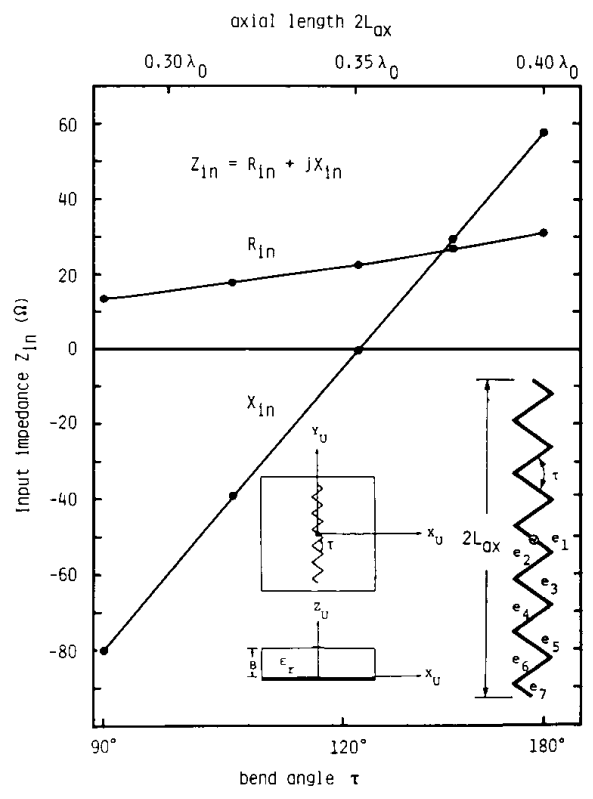

Fig. 4. Input impedance of zigzag dipole antenna versus bend angle.
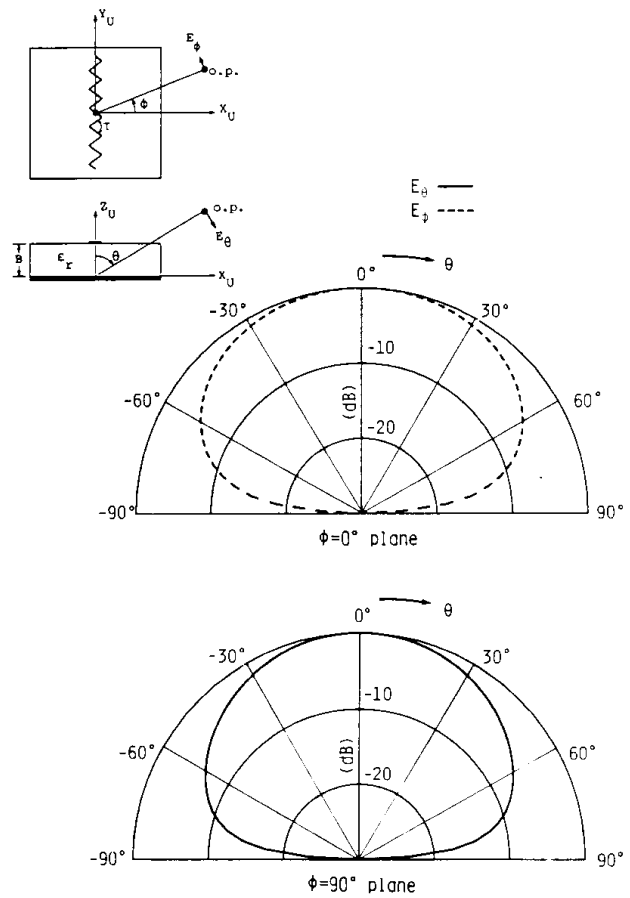

Fig. 5. Radiation pattern of zigzag dipole antenna with bend angle of $\tau=$ $122.5^{\circ}$.

dipole, which is defined as $\mathrm{SR}=\left(\left(\lambda_{0} / 2\right)-\right.$ axial length $\left.2 L_{\text {ax }}\right) /\left(\lambda_{0} / 2\right)$, is calculated to have a value 0.30 at resonance. The radiation pattern for this case is shown in Fig. 5, in which the cross polarization is suppressed under $-45 \mathrm{~dB}$.

A loop antenna is also a resonant-type antenna. Fig. 6 shows the current distribution when the loop resonates at a loop circumference of $0.8 \lambda_{0}$, where the resonance impedance is

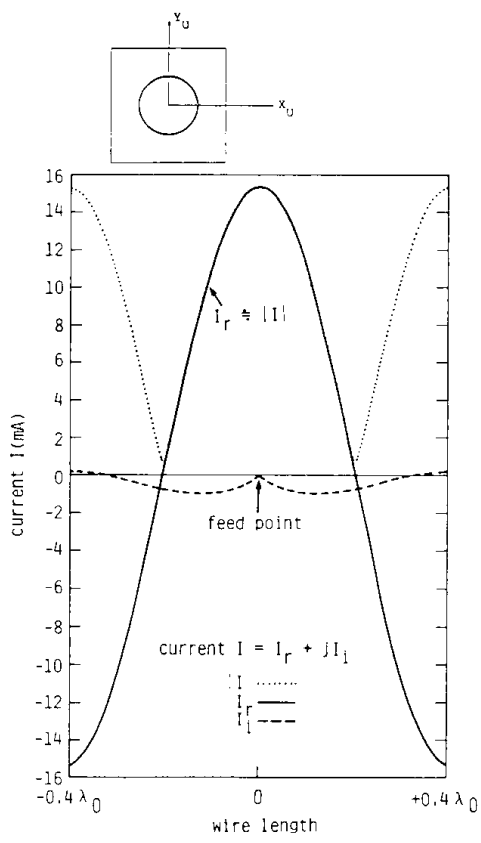

Fig. 6. Current distribution of loop antenna with circumference of $0.8 \lambda_{0}$.

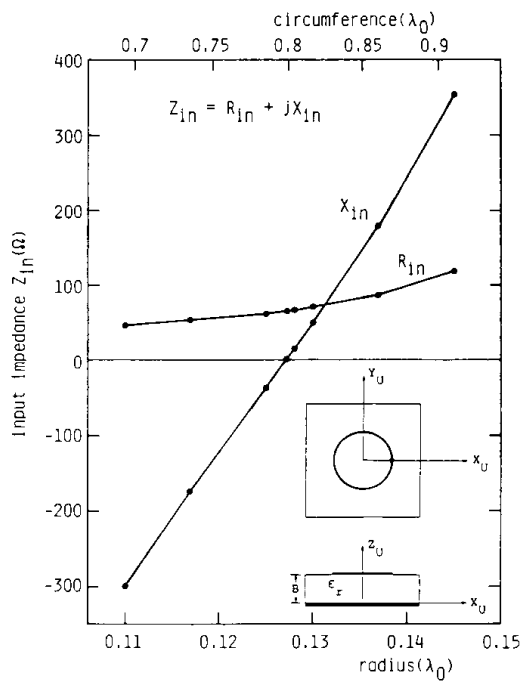

Fig. 7. Input impedance of loop antenna versus loop radius

evaluated to be $65 \Omega$. An overview of the impedance variation with loop radius is shown in Fig. 7. As in the previous case, the resistance curve indicates a slow change when compared with the reactance curve. Fig. 8 is the radiation pattern of the loop at resonance. The half-power beam width is calculated to be $\pm 41^{\circ}$.

Finally, we show the numerical results for the round spiral, which is a nonresonant type antenna. The antenna arm is defined by the Archimedean spiral function $r=A \phi_{s}(A$ is the spiral constant, $\phi_{s}$ the winding angle) shown in the upper illustration of Fig. 9. The typical current distributions and their phase progressions are shown in Figs. 9(a) and 9(b). Since 

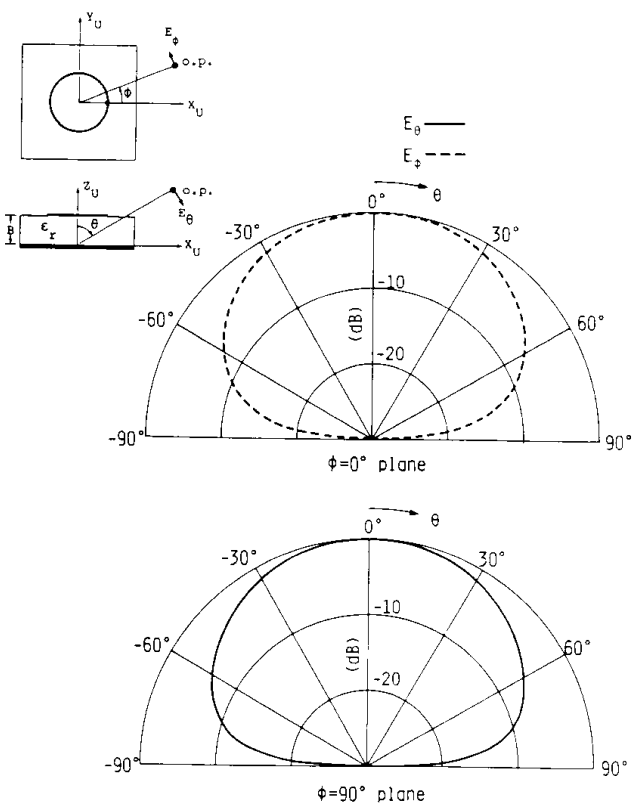

Fig. 8. Radiation pattern of loop antenna with circumference of $0.8 \lambda_{0}$.
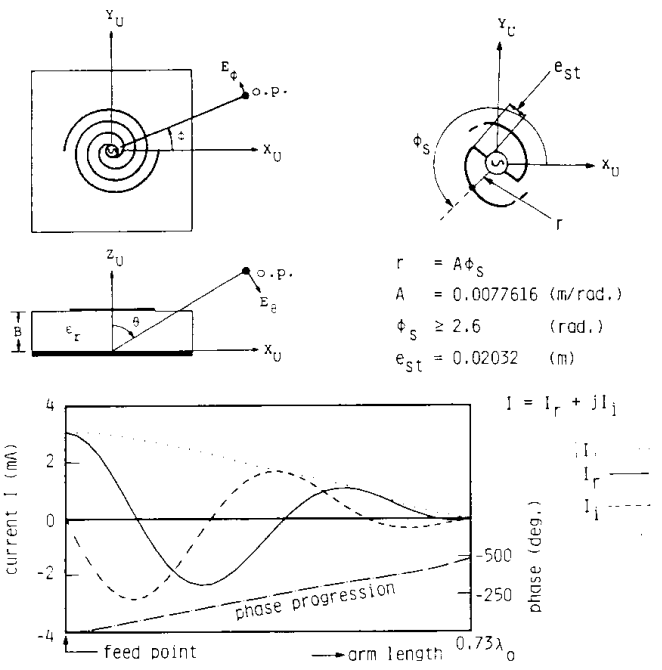

(a)

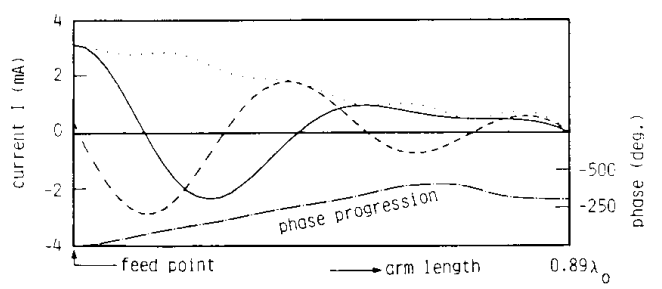

(b)

Fig. 9. Current distribution and phase progression of spiral antenna.

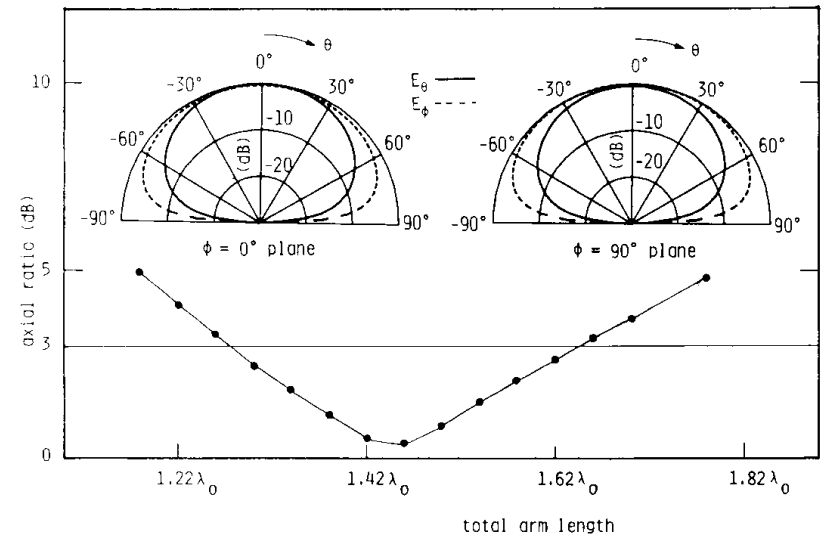

Fig. 10. Axial ratio and radiation pattern of spiral antenna.

these are symmetrical with respect to the feed point, only half of the currents and half of the phase progressions are shown in these figures. The smoother decay of the current shown in Fig. 9 (a) generates a better radiation field of circular polarization. This is confirmed in the axial ratio of Fig. 10, together with the radiation pattern for a total arm length of $1.46 \lambda_{0}(=2 \times$ $0.73 \lambda_{0}$ ). Also note that the smoothly decaying current contributes to realizing an impedance of approximately pure resistance as shown in Fig. 11.

\section{CONCLUSION}

The formulation for a printed wire antenna on a grounded dielectric substrate is made to obtain the current distribution. The printed wire arm is arbitrary in shape and subdivided into many segments which are regarded as being linear. For application of the moment method to the antenna, the current is expanded using piecewise sinusoidal functions. Introducing 


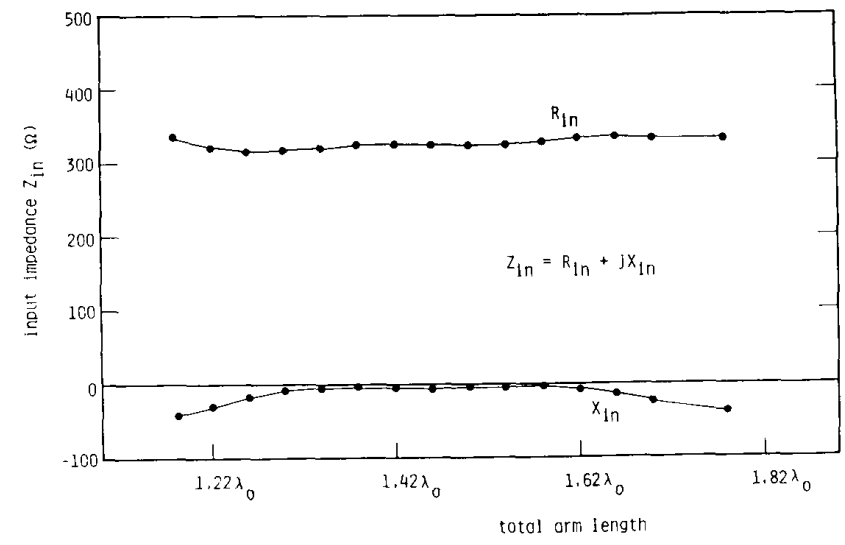

Fig. 11. Input impedance of spiral antenna.

local coordinate systems leads to a simple expression for the tangential field at an observation point due to the current on each wire segment. The impedance matrix element $Z_{m, n}$ is constructed using weighting functions of piecewise sinusoids.

An application of the stationary phase method simplifies the expression for the far-zone radiation field, which is evaluated on the basis of the coefficients of the expansion functions. The evaluation does not include any calculations of integrals or derivatives. As a result, less computer time is required.

The described techniques are used to analyze three antennas; they are a zigzag dipole antenna, a loop antenna, and a round spiral antenna. The analyses give informative data on the current distribution, the radiation pattern, and the input impedance. It is demonstrated that changing the bend angle, or changing the loop radius, can result in an impedance of pure resistance. The numerical results for the spiral show that it radiates a circularly polarized wave, due to a smoothly decaying current distribution.

\section{REFERENCES}

[1] R. C. Johnson and H. Jasik, Eds., Antenna Engineering Handbook. New York: McGraw-Hill, 1984, chs. 4 and 5.

[2] W. L. Stutzman and G. A. Thiele, Antenna Theory and Design. New York: Wiley, 1981, p. 310.

[3] K. K. Mei, "On the integral equations of thin wire antennas," IEEE Trans. Antennas Propagat., vol. AP-13, pp. 374-378, May 1965.

[4] N. K. Uzunoglu, N. G. Alexopoulos, and J. G. Fikioris, "Input and mutual impedance computation for microstrip dipole antennas," in Proc. 1979 Int. Symp. Digest Antennas Propagat., Seattle, WA, pp. 374-378.

[5] —-, "Radiation properties of microstrip dipoles," IEEE Trans. Antennas Propagat., vol. AP-27, pp. 853-858, Nov. 1979. (See also correction, IEEE Trans. Antennas Propagat., vol. AP-30, p. 526, May 1982.)

[6] D. M. Pozar, "Input impedance and mutual coupling of rectangular microstrip antennas," IEEE Trans. Antennas Propagat., vol. AP-30, pp. 1191-1196, Nov. 1982

[7] I. E. Rana and N. G. Alexopoulos, "Printed wire antennas," in Proc. Workshop on Printed Circuit Antenna Technology, Las Cruces, NM, Oct. 1979, pp. 30-1-30-14.

[8] I. E. Rana, N. G. Alexopoulos, and P. B. Katehi, "Theory of microstrip Yagi-Uda arrays," Radio Sci., vol. 16, no. 6, pp. 10771079, Nov.-Dec. 1981.

[9] J. R. Mosig and F. E. Gardiol, "The near field of an open microstrip structure," in IEEE Int. Antennas Propagat. Soc, Symp. Dig., pp. $379-382,1979$
[10] A. J. M. Soares, S. B. A. Fonesca, and A. J. Giarola, "The effect of a dielectric cover on the current distribution and input impedance of printed dipoles," IEEE Trans. Antennas Propagat., vol. AP-32, pp. 1149-1153, Nov. 1984.

[11] I. E. Rana and N. G. Alexopoulos, "Current distribution and input impedance of printed dipoles," IEEE Trans. Antennas Propagat., vol. AP-29, pp. 99-105, Jan. 1981.

[12] H. Nakano, K. Nogami, S. Arai, H. Mimaki, and J. Yamauchi, "A spiral antenna backed by a conducting plane reflector," IEEE Trans. Antennas Propagat., vol. AP-34, pp. 791-796, June 1986.

[13] D. R. Jackson and N. G. Alexopoulos, "An asymptotic extraction technique for evaluating Sommerfeld-type integrals," IEEE Trans. Antennas Propagat., vol. AP-34, pp. 1467-1470, Dec. 1986.

[14] R. H. Clarke and J. Brown, Diffraction Theory and Antennas. New York: Wiley, 1980.

[15] H. Nakano, H. Tagami, A. Yoshizawa, and J. Yamauchi, "Shortening ratios of modified dipole antennas," IEEE Trans. Antennas Propagat., vol. AP-32, pp. 385-387, Apr. 1984.

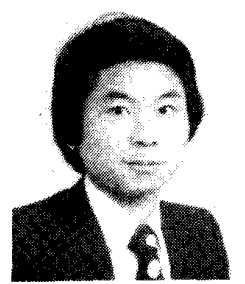

Hisamatsu Nakano (M'75-SM'87) was born in Ibaraki, Japan, on April 13, 1945. He received the B.E., M.E., and Dr.E. degrees in electrical engineering from Hosei University, Tokyo, Japan, in 1968,1970 , and 1974, respectively.

Since 1973, he has been a member of the Faculty of Hosei University, where he is now a Professor of Electrical Engineering. His research interests are numerical methods for microwave antennas, and scattering problems. In 1981 he was a Visiting Professor at Syracuse University, Syracuse, NY, and contributed to formulating an electromagnetic coupling between a wire and a slot. In 1986 and 1987 he was a Visiting Professor at the University of Manitoba, Winnipeg, MB, Canada, and the University of California, Los Angeles, respectively.

Dr. Nakano received the International Scientific Exchange Award in 1986 and the Best Paper Award of the IEE Fifth International Conference on Antennas and Propagation in 1987. He is a member of the Institute of Electronics, Information and Communication Engineers of Japan.

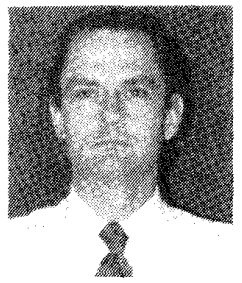

Stephen R. Kerner (S'83) was born in El Paso, TX on January 16,1948 . He received the B.S. degree in physics from the California State University, Northridge, and the M.S. degree in engineering from the University of California, Los Angeles, where he is currently working toward the Ph.D degree in electrical engineering.

He has worked for the Hughes Aircraft Company, Radar Systems Group, since September 1977. 


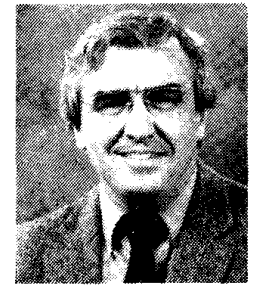

Nicólaos G. Alexopoulos (S'68-M'69-SM'82F'87) graduated from the 8th Gymnasium of Athens, Greece, and received the B.S.E.E., M.S.E.E., and Ph.D. degrees from the University of Michigan, Ann Arbor, in 1964, 1967, and 1968, respectively.

Currently he is Professor and Chairman of the Electrical Engineering Department at the University of California, Los Angeles. He is also a Consultant with Northrop Corporation's Advanced Systems Division. His current research interests are in electromagnetic theory as it applies to the modeling of integrated-circuit components and printed circuit antennas for microwave and millimeter-wave applications, substrate materials and their effect on integrated-circuit structures and printed antennas, integrated-circuit antenna arrays, and antenna scattering studies. He is furthermore interested in the interaction of electromagnetic waves with materials and, in particular, active media.

Dr. Alexopoulos is the Associate Editor of the Electromagnetics Journal and Alta Frequenza and is on the Editorial Boards of the IEEE TRANSACTIONS ON MICROWAVE THEORY AND TECHNIQUES and the International Journal on Electromagnetics Theory. He served as the 1974 Chairman of the IEEE APS Chapter. He is the corecipient of the 1983 IEEE APS R. W. P. King Best Paper Award and of the 1984 IEEE Antennas and Propagation Society's S. A. Schelkunoff Transactions Prize Paper Award. 